\title{
Fatores associados ao letramento funcional em saúde de mulheres atendidas pela Estratégia de Saúde da Família
}

\section{Factors associated with the functional health literacy in women served by the Family Health Strategy}

\author{
Angélica Atala Lombelo Campos ${ }^{1 *}$ (1) , Felipe Silva Neves ${ }^{1,2}$ (D), Raphael de Freitas Saldanha ${ }^{3}$ (D), \\ Kristiane de Castro Dias Duque', Maximiliano Ribeiro Guerra', Isabel Cristina Gonçalves Leite', \\ Maria Teresa Bustamante Teixeira'
}

\begin{abstract}
'Departamento de Saúde Coletiva, Faculdade de Medicina, Universidade Federal de Juiz Fora (UFJF) - Juiz de Fora (MG), Brasil. 2Departamento de Nutrição, Instituto de Ciências Biológicas, Universidade Federal de Juiz de Fora (UFJF) - Juiz de Fora (MG), Brasil.

${ }^{3}$ Instituto de Comunicação e Informação Científica e Tecnológica em Saúde, Fundação Oswaldo Cruz (FIOCRUZ) - Rio de Janeiro (RJ), Brasil.
\end{abstract}

\begin{abstract}
Como citar: Campos AAL, Neves FS, Saldanha RF, Duque KCD, Guerra MR, Leite ICG, et al. Fatores associados ao letramento funcional em saúde de mulheres atendidas pela Estratégia de Saúde da Família. Cad Saúde Colet, 2020;28(1):66-76. https://doi.org/10.1590/1414-462X202000280295
\end{abstract}

\section{Resumo}

Introdução: O letramento funcional em saúde (LFS) diz respeito à habilidade dos indivíduos em compreender as informações relacionadas à saúde e está relacionado a diversos desfechos de saúde. Objetivo: Investigar a associação do LFS com fatores sociodemográficos, apoio social, autoavaliação do estado de saúde e perfil de acesso aos serviços de saúde em mulheres assistidas pela Estratégia de Saúde da Família (ESF). Método: Estudo transversal, conduzido em 2015-2016, em duas Unidades de Atenção Primária à Saúde cobertas pela ESF, de um município da região Sudeste do Brasil. A amostra foi composta por 439 mulheres, entre 25 e 64 anos. O LFS foi avaliado por meio do Brief Test of Functional Health Literacy in Adults (B-TOFHLA). Efetuaram-se cálculos da razão de prevalência (RP). Posteriormente, construiu-se um modelo de regressão de Poisson de variância robusta, sendo admitida significância estatística quando $p$ $\leq 0,05$. Resultados: Foi constatado que $53,5 \%$ das mulheres apresentaram um baixo LFS, o qual associou-se à idade superior aos 40 anos ( $\mathrm{RP}=1,18 ; \mathrm{IC} 95 \%$ : 1,07-1,31), ao grau de instrução inferior ao ensino médio completo ( $R P=1,26$; IC 95\%: 1,15-1,38), à baixa renda ( $R P=1,13 ;$ IC 95\%: 1,04-1,23) e à autodeclaração da cor parda ou preta (RP=1,06; IC 95\%: 1,01-1,12). Conclusão: Os resultados acentuaram a importância do LFS como estratégia para a abordagem de populações com maior vulnerabilidade socioeconômica.

Palavras-chave: mulheres; alfabetização em saúde; fatores socioeconômicos; atenção primária à saúde.

\section{Abstract}

Background:' Functional health literacy (FHL) concerns the ability of individuals to understand health-related information, which is related to various health outcomes. Objective: Investigate the association between FHL and socio-demographic factors, social support, self-assessment of health status and access to health services in women assisted by the Family Health Strategy (FHS). Method: A cross-sectional study, conducted in 2015-2016, in two Primary Health Care Units covered by the FHS of a municipality in the Southeast region of Brazil. The sample consisted of 479 participants, aged between 24 and 64 years. FHL was evaluated by the Brief Test of Functional Health Literacy in Adults (B-TOFHLA). Prevalence Ratio (PR), with Confidence Interval $(\mathrm{Cl})$ of $95 \%$, was performed. Subsequently, a Poisson regression model of robust variance was constructed, and statistical significance was accepted when

Trabalho realizado nas duas Unidades de Atenção Primária à Saúde - Juiz de Fora (MG), Brasil. Correspondência: Angélica Atala Lombelo Campos. E-mail: angelica.atala@hotmail.com Conflito de interesses: nada a declarar.

Recebido em: Jun 18, 2018. Aprovado em: Abr 05, 2019
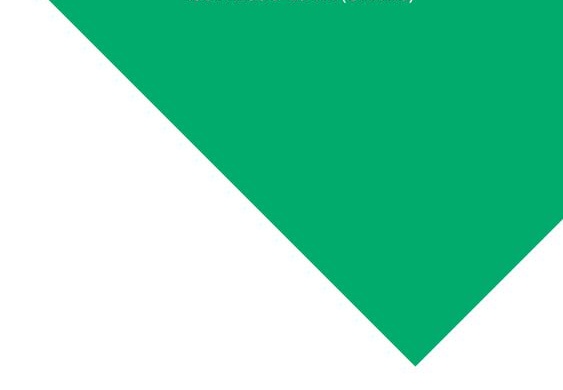
$\mathrm{p} \leq 0.05$. Results: It was found that $53.5 \%$ of the women had a low $\mathrm{FHL}$, which was associated with age over $40(\mathrm{RP}=1.18,95 \% \mathrm{Cl}: 1.07-1.31)$, low education level $(\mathrm{RP}=1.26,95 \% \mathrm{Cl}$ : 1.15-1.38), low income $(\mathrm{RP}=1.13,95 \% \mathrm{Cl} 1.04-1.23)$ and self-declaration brown or black skin color ( $\mathrm{PR}=1.06,95 \% \mathrm{Cl} 1.01-1.12)$. Conclusion: The results reinforce the importance of $\mathrm{FHL}$ use as a strategy for the management of populations with greater socioeconomic vulnerability.

Keywords: women; health literacy; socioeconomic factors; primary health care.

\section{INTRODUÇÃO}

O letramento funcional em saúde (LFS) diz respeito ao nível de compreensão de informações imprescindíveis para se tomar decisões no âmbito da saúde. Dessa forma, contribui para fomentar transformações de cunho individual, cultural, social, econômico e político ${ }^{1,2}$.

Tal constructo considera que indivíduos mesmo sabendo ler e escrever podem apresentar incapacidade para compreender e interpretar os esclarecimentos que lhes são concedidos por profissionais da saúde. Sendo assim, o LFS requer uma sucessão de competências cognitivas, respectivamente: (a) acesso - habilidade para procurar, encontrar e obter informações sobre saúde; (b) compreensão - habilidade para compreender as informações acessadas; (c) avaliação - habilidade para interpretar, filtrar, julgar e avaliar as informações acessadas; (d) aplicação - habilidade para comunicar e usar as informações na tomada de decisões para a manutenção ou melhora da saúde².

O entendimento dos usuários quanto às observações repassadas pelos profissionais é determinante não só para a adesão ao que é prescrito mas também para o sucesso do plano de cuidados e adoção de hábitos de vida saudáveis. Porém, muitas vezes existe um desencontro entre o que é dito no serviço de saúde e o que é realmente compreendido pelos usuários ${ }^{3,4}$.

Uma comunicação acessível, além do vínculo entre pacientes e profissionais, é indispensável para a continuidade do cuidado e resolutividade da Atenção Primária à Saúde, considerada a principal porta de entrada no sistema de saúde, sendo responsável pelo ordenamento dos usuários na Rede de Atenção à Saúde 5 .

No Brasil, investigações sobre o LFS são recentes e ainda não foi traçado um panorama nacional sobre essa temática. $\mathrm{O}$ crescente interesse no assunto se justifica pelo fato de que baixos níveis de LFS resultam em piores desfechos clínicos e, consequentemente, em maiores gastos para o sistema de saúde. Portanto, o objetivo deste estudo foi investigar a associação do LFS com fatores sociodemográficos, apoio social, autoavaliação do estado de saúde e perfil de acesso aos serviços de saúde em mulheres assistidas pela Estratégia de Saúde da Família.

\section{MÉTODO}

\section{Delineamento do estudo}

Trata-se de um estudo transversal inserido na coorte intitulada "Avaliação de estratégias para o rastreamento do câncer do colo do útero em mulheres cobertas pela Estratégia de Saúde da Família após cinco anos de seguimento no município de Juiz de Fora, Minas Gerais" (região Sudeste do Brasil).

A pesquisa originou-se de uma colaboração entre o Núcleo de Assessoria, Treinamento e Estudos em Saúde da Universidade Federal de Juiz de Fora (NATES/UFJF), o Instituto de Medicina Social da Universidade do Estado do Rio de Janeiro (IMS/UERJ) e o Instituto Nacional de Câncer José Alencar Gomes da Silva (INCA), cuja primeira fase da coleta de dados foi realizada em 2010-2012 ${ }^{6}$. A atual investigação é relativa à segunda fase, conduzida no período de dezembro de 2015 a outubro de 2016.

Em conformidade com as questões éticas, foi obtida a aprovação junto ao Comitê de Ética em Pesquisa do IMS/UERJ (parecer: 1.323.441 - CAAE: 48067815.2.0000.5260), sendo requisitadas as assinaturas das participantes em Termo de Consentimento Livre e Esclarecido. 


\section{Participantes}

A amostra foi selecionada a partir de 778 mulheres, entre 20 e 59 anos, residentes na área de abrangência de duas Unidades de Atenção Primária à Saúde (UAPS) cobertas pela Estratégia de Saúde da Família, que participaram da primeira fase de seguimento ${ }^{6}$. Para a segunda fase, foram elegíveis participantes entre 25 e 64 anos que ainda viviam em endereços na área de abrangência das mesmas UAPS, excluindo-se as histerectomizadas e as que estavam gestantes no período da coleta de dados. Agentes comunitários de saúde, juntamente com pesquisadores do NATES/UFJF, analisaram a listagem de participantes da primeira fase da coorte e conduziram as etapas de identificação e elegibilidade da amostra.

Ademais, atendendo aos protocolos do instrumento de avaliação do LFS - Brief Test of Functional Health Literacy in Adults (B-TOFHLA) -, foram estabelecidos os seguintes critérios de inclusão: não estar sob a custódia da justiça; saber ler ou ter pelo menos um ano de estudo completo; apresentar acuidade visual adequada de acordo com a escala de sinais de Snellen ${ }^{7}$, com capacidade de leitura até a linha 20/50; não relatar enfermidade psiquiátrica ou neurológica de efeitos cognitivos.

A Figura 1 ilustra o diagrama de fluxo acerca das etapas de identificação, elegibilidade e inclusão das participantes. Das 778 mulheres submetidas às avaliações em 2010-2012, não foi possível recrutar 226, pois três faleceram, 201 passaram a residir em endereços fora da área de abrangência das UAPS, 12 estavam gestantes no período da nova coleta de dados e 10 foram submetidas à histerectomia no transcorrer da primeira para a segunda fase do estudo, resultando 552 mulheres elegíveis para avaliação. Ressalta-se que as gestantes e as histerectomizadas foram excluídas porque a coorte da qual originou-se o presente estudo também se destinava a avaliar o rastreamento para o câncer do colo do útero.

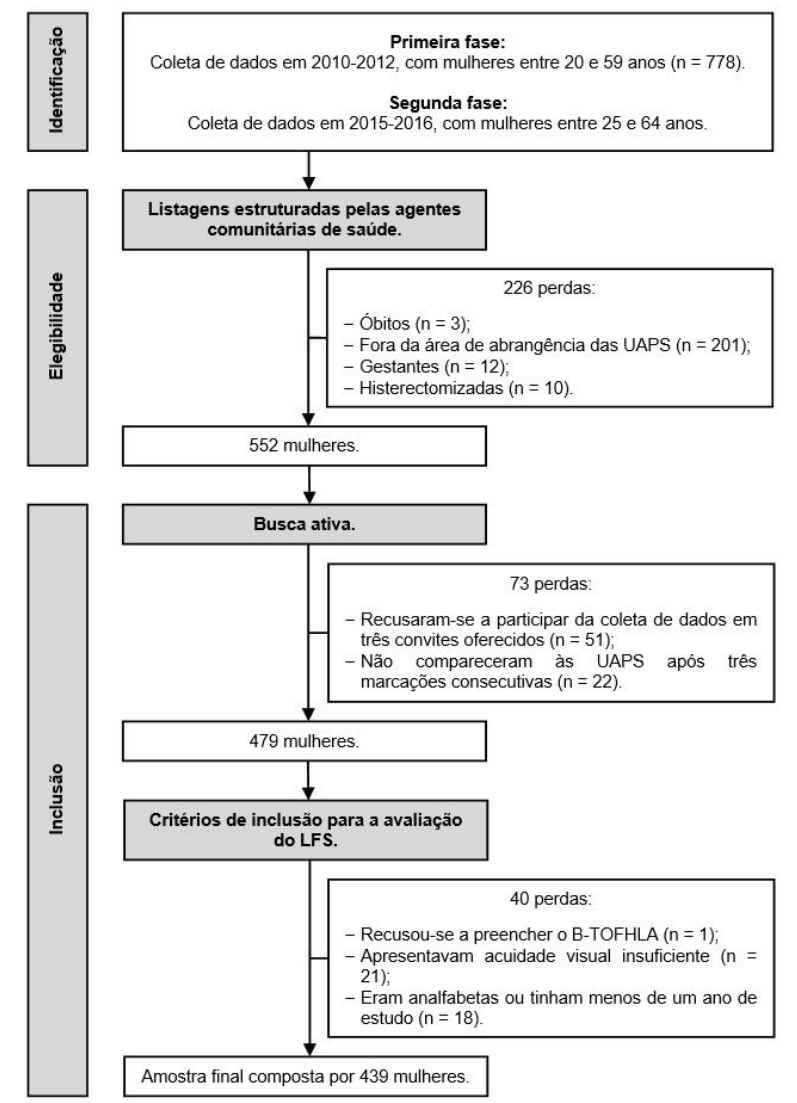

Figura 1. Diagrama de fluxo acerca das etapas de recrutamento e composição da amostra do estudo, Juiz de Fora/MG, 2015-2016. B-TOFHLA: Brief Test of Functional Health Literacy in Adults; LFS: letramento funcional em saúde; UAPS: Unidades de Atenção Primária à Saúde 
Posteriormente, foram contabilizadas mais 73 perdas, pois 51 mulheres se recusaram a participar da coleta de dados após três convites consecutivos, realizados em dias e horários alternados, e 22 não compareceram ao recrutamento mesmo tendo confirmado a presença em, pelo menos, três agendamentos por contato telefônico em diferentes dias e horários.

Enfim, das 479 participantes avaliadas, somaram-se 40 perdas devido aos critérios de inclusão para avaliação do LFS por meio do B-TOFHLA: uma se recusou a preencher o instrumento e 39 não atendiam aos pré-requisitos necessários (21 apresentavam acuidade visual insuficiente e 18 eram analfabetas ou tinham menos de um ano de estudo completo).

\section{Coleta de dados}

Para a coleta de dados, uma equipe treinada de profissionais da saúde administrou um questionário por meio de entrevista individual, face a face, com preenchimento eletrônico no aplicativo ODK Collect (versão 1.5.0, Open Data Kit, EUA), abordando aspectos sociodemográficos, apoio social, autoavaliação do estado de saúde e perfil de acesso aos serviços de saúde.

Acrescenta-se que para avaliar o LFS utilizou-se o questionário B-TOFLHA, que é composto por uma etapa autoaplicável (avaliação da leitura) e outra parte aplicada por um profissional devidamente treinado (avaliação do numeramento).

\section{Variáveis do estudo}

Determinou-se como variável dependente o nível de LFS avaliado por meio do B-TOFHLA, o qual foi desenvolvido por Baker et al. ${ }^{8}$ e, posteriormente, foi traduzido e validado no Brasil por Carthery-Goulart et al. ${ }^{9}$. Este instrumento foi escolhido por apresentar uma abordagem mais ampla, que extrapola a habilidade para o reconhecimento de palavras ou a simples compreensão de um texto ${ }^{10}$; ele possibilita avaliar a capacidade de leitura por meio de 36 itens e inclui outros quatro tópicos para a análise do numeramento, levando cerca de 12 minutos para ser aplicado. A pontuação total é de 100 pontos, sendo 72 referentes à leitura (peso 2 para cada item) e 28 referentes ao numeramento (peso 7 para cada item). Conforme o valor total obtido, classifica-se o respondente em três níveis de LFS: inadequado (de 0 a 53 pontos), marginal (de 54 a 66 pontos) ou adequado (de 67 a 100 pontos) 8,9. $^{8}$. Neste estudo, foi atribuído um “nível baixo" às participantes com classificação inadequada ou marginal.

O B-TOFHLA reproduz situações rotineiras encontradas por usuários dos serviços de saúde. A compreensão da leitura aborda instruções acerca da preparação individual para um exame de raios $X$ do estômago, bem como direitos e responsabilidades do usuário do sistema de saúde. A cada cinco ou seis palavras há uma lacuna que deve ser preenchida com a melhor opção, dentre uma lista de quatro palavras, completando, assim, o sentido da frase. Para avaliar o numeramento, são apresentados um rótulo de comprimidos, um resultado de exame e um cartão de agendamento de consulta através dos quais os usuários devem interpretar a prescrição de medicação, o nível glicêmico e a data de marcação de consulta clínica ${ }^{8,9}$.

As variáveis independentes foram agrupadas em dois blocos:

(a) Primeiro bloco (perfil sociodemográfico) - faixa etária; situação conjugal; grau de instrução; cor da pele autodeclarada; assiduidade religiosa (comparecer a cultos ou atividades religiosas pelo menos duas vezes ao mês); situação ocupacional; situação socioeconômica segundo os critérios da Associação Brasileira de Empresas de Pesquisa (ABEP) ${ }^{11}$; renda per capita; ser beneficiária ou não do Programa Bolsa Família.

(b) Segundo bloco (apoio social, autoavaliação do estado de saúde e perfil de acesso aos serviços de saúde) - apoio social (sentir-se à vontade para conversar com pelo menos um amigo ou familiar); autoavaliação do estado de saúde; acesso aos serviços de saúde da UAPS nos últimos 12 meses; recebimento de visita domiciliar; posse de plano de saúde particular; participação em grupo de direitos sexuais e reprodutivos.

\section{Análises estatísticas}

Inicialmente, os dados foram submetidos à análise descritiva (frequências, proporções e medidas de tendência central) para a caracterização da amostra. Em seguida, na análise 
bivariada utilizou-se o teste qui-quadrado de Pearson para verificar a associação do desfecho com as variáveis independentes. A análise multivariada foi realizada por meio de regressão de Poisson robusta, com o intuito de verificar a permanência da força de associação mediante o controle de variáveis de confusão. O modelo final foi concebido a partir do método Backward, sendo incluídas todas as variáveis que apresentaram $p \leq 0,20$. A medida estimada foi a razão de prevalência (RP) e seus respectivos intervalos de confiança de 95\% (IC 95\%), permanecendo no modelo final aquelas variáveis que mantiveram um valor $p \leq 0,05$. As análises estatísticas foram conduzidas no software $\operatorname{STATA}^{\circ}$ (versão 13.0; StataCorp. LP, EUA).

\section{RESULTADOS}

Foram incluídas na análise 439 mulheres, com média de idade de 42,5 anos $( \pm 10,6)$. Grande parte da amostra referiu grau de instrução inferior ao ensino fundamental completo $(44,2 \%)$ e, em média, 8 anos completos de estudo. A maioria tinha sido casada $(75,4 \%)$, trabalhava $(60,1 \%)$, pertencia à religião católica $(46,9 \%)$, frequentava cultos ou atividades religiosas ao menos duas vezes por semana $(75,6 \%)$, autodeclarou-se preta ou parda $(61,7 \%)$ e pertencia à classe econômica baixa (83,5\%). Além disso, 78,1\% não recebia Bolsa Família e 74,7\% não tinha plano de saúde particular.

O baixo LFS foi identificado em $53,5 \%$ das mulheres avaliadas, estando diretamente associado à idade (RP = 1,26; IC 95\%: 1,13-1,39), com valores crescentes de RP conforme a progressão da faixa etária, e inversamente associado ao grau de instrução $(R P=1,45 ; 1 C v, 56)$, com valores decrescentes de RP conforme o avanço da escolaridade. Tanto a baixa renda segundo os critérios da $A B E P(R P=1,24 ;$ IC $95 \%: 1,13-1,35)$ quanto a renda per capita menor que meio salário mínimo ( $R P=1,09 ;$ IC $95 \%: 1,03-1,16)$ foram associadas ao baixo $L F S$. Por outro lado, observou-se menor probabilidade de baixo LFS em mulheres que nunca foram casadas $(\mathrm{RP}=0,87 ;$ IC 95\%: 0,81-0,94) (Tabela 1).

Tabela 1. Perfil sociodemográfico de mulheres atendidas pela Estratégia de Saúde da Família segundo o nível de letramento funcional em saúde (LFS), Juiz de Fora, MG, 2015-2016

\begin{tabular}{|c|c|c|c|c|c|}
\hline \multirow[b]{2}{*}{ Variável } & \multicolumn{5}{|c|}{ Nível de LFS } \\
\hline & $n *$ & $\begin{array}{c}\text { Adequado } \\
\text { n (\%) }\end{array}$ & $\begin{array}{l}\text { Baixo } \\
\text { n (\%) }\end{array}$ & $\begin{array}{c}\text { RP } \\
\text { (IC 95\%) }\end{array}$ & $\mathbf{p}$ \\
\hline \multicolumn{6}{|l|}{ Faixa etária } \\
\hline 25 a 29 anos & 59 & $45(76,3)$ & $14(23,7)$ & 1 & \\
\hline 30 a 39 anos & 124 & $78(62,9)$ & $46(37,1)$ & $1,11(0,99-1,23)$ & 0,062 \\
\hline 40 a 49 anos & 129 & $57(44,2)$ & $72(55,8)$ & $1,26(1,13-1,39)$ & $<0,001$ \\
\hline 50 a 59 anos & 100 & $21(21,0)$ & $79(79,0)$ & $1,44(1,31-1,59)$ & $<0,001$ \\
\hline$\geq 60$ anos & 27 & $3(11,1)$ & $24(88,9)$ & $1,52(1,37-1,70)$ & $<0,001$ \\
\hline \multicolumn{6}{|l|}{ Situação conjugal } \\
\hline Foi casada & 331 & $138(41,7)$ & $193(58,3)$ & 1 & \\
\hline Nunca foi casada & 107 & $66(61,7)$ & $41(38,3)$ & $0,87(0,81-0,94)$ & $<0,001$ \\
\hline
\end{tabular}

LFS: letramento funcional em saúde; RP: razão de prevalência; IC: intervalo de confiança. * As oscilações do tamanho da amostra são justificadas pela ausência de informação. ${ }^{\dagger}$ Comparecer a cultos ou atividades religiosas pelo menos duas vezes ao mês. ${ }^{\S}$ Renda média/alta: classes A, B1 e B2; renda baixa: classes C1, C2 e D/E. Renda média/alta: renda per capita $\geq 0,5$ salário mínimo. 
Tabela 1. Continuação...

\begin{tabular}{|c|c|c|c|c|c|}
\hline \multirow[b]{2}{*}{ Variável } & \multicolumn{5}{|c|}{ Nível de LFS } \\
\hline & $\mathbf{n} *$ & $\begin{array}{c}\text { Adequado } \\
\text { n (\%) }\end{array}$ & $\begin{array}{l}\text { Baixo } \\
\text { n (\%) }\end{array}$ & $\begin{array}{c}\text { RP } \\
\text { (IC 95\%) }\end{array}$ & $\mathbf{p}$ \\
\hline \multicolumn{6}{|l|}{ Grau de instrução } \\
\hline Ensino médio completo ou mais & 137 & $106(77,4)$ & $31(22,6)$ & 1 & \\
\hline $\begin{array}{l}\text { Ensino fundamental completo ou } \\
\text { médio incompleto }\end{array}$ & 194 & $75(38,7)$ & $119(61,3)$ & $1,31(1,22-1,41)$ & $<0,001$ \\
\hline $\begin{array}{l}\text { Analfabeta ou ensino } \\
\text { fundamental incompleto }\end{array}$ & 108 & $23(21,3)$ & $85(78.7)$ & $1,45(1,35-1,56)$ & $<0,001$ \\
\hline \multicolumn{6}{|l|}{ Cor da pele autodeclarada } \\
\hline Branca & 156 & $78(50,0)$ & $78(50,0)$ & 1 & \\
\hline Parda/preta & 271 & $119(43,9)$ & $152(56,1)$ & $1,04(0,97-1,11)$ & 0,228 \\
\hline \multicolumn{6}{|l|}{ Assiduidade religiosa ${ }^{+}$} \\
\hline Sim & 332 & $153(46,1)$ & $179(53,9)$ & 1 & \\
\hline Não & 105 & $50(47,6)$ & $55(52,4)$ & $0,99(0,92-1,06)$ & 0,784 \\
\hline \multicolumn{6}{|l|}{ Situação ocupacional } \\
\hline Trabalha & 264 & $124(47,0)$ & $140(53,0)$ & 1 & \\
\hline Não trabalha & 169 & $77(45,6)$ & $92(54,4)$ & $1,01(0,94-1,07)$ & 0,774 \\
\hline \multicolumn{6}{|l|}{ Situação socioeconômica ${ }^{\S}$} \\
\hline Média/alta & 75 & $54(72,0)$ & $21(28,0)$ & 1 & \\
\hline Baixa & 364 & $150(41,2)$ & $214(58,8)$ & $1,24(1,13-1,35)$ & $<0,001$ \\
\hline \multicolumn{6}{|l|}{ Renda per capita $"$} \\
\hline Média/alta & 238 & $126(52,9)$ & $112(47,1)$ & 1 & \\
\hline Baixa & 195 & $76(38,9)$ & $119(61,1)$ & $1,09(1,03-1,16)$ & 0,003 \\
\hline \multicolumn{6}{|c|}{ Beneficiária do Programa Bolsa Família } \\
\hline Não & 343 & $158(46,1)$ & $185(53,9)$ & 1 & \\
\hline Sim & 95 & $46(48,4)$ & $49(51,6)$ & $0,98(0,91-1,06)$ & 0,686 \\
\hline
\end{tabular}

LFS: letramento funcional em saúde; RP: razão de prevalência; IC: intervalo de confiança. * As oscilações do tamanho da amostra são justificadas pela ausência de informação. ${ }^{\dagger}$ Comparecer a cultos ou atividades religiosas pelo menos duas vezes ao mês. ${ }^{5}$ Renda média/alta: classes A, B1 e B2; renda baixa: classes C1, C2 e D/E. Renda média/alta: renda per capita $\geq 0,5$ salário mínimo.

As participantes que não dispunham de apoio social ( $R P=1,06$; IC 95\%: 1,01-1,13), as que tinham percepção negativa da própria saúde $(\mathrm{RP}=1,10 ; \mathrm{IC} 95 \%: 1,02-1,18)$ e as que não tinham plano de saúde particular ( $\mathrm{RP}=1,10$; IC 95\%: 1,02-1,18) exibiram maiores probabilidades de terem baixo LFS (Tabela 2). 
Tabela 2. Apoio social, autoavaliação do estado de saúde e perfil de acesso aos serviços de saúde de mulheres atendidas pela Estratégia de Saúde da Família segundo o nível de letramento funcional em saúde (LSF), Juiz de Fora, MG, 2015-2016

\begin{tabular}{|c|c|c|c|c|c|}
\hline \multirow{2}{*}{ Variável } & \multicolumn{5}{|c|}{ Nível de LFS } \\
\hline & $\mathbf{n} *$ & $\begin{array}{c}\text { Adequado } \\
\text { n (\%) }\end{array}$ & $\begin{array}{l}\text { Baixo } \\
\text { n (\%) }\end{array}$ & $\begin{array}{c}\text { RP } \\
\text { (IC 95\%) }\end{array}$ & $\mathbf{p}$ \\
\hline \multicolumn{6}{|l|}{ Apoio social $^{+}$} \\
\hline Sim & 272 & $137(50,4)$ & $135(49,6)$ & 1 & \\
\hline Não & 167 & $37(40,1)$ & $100(59,9)$ & $1,06(1,01-1,13)$ & 0,034 \\
\hline \multicolumn{6}{|c|}{ Autoavaliação do estado de saúde } \\
\hline Positiva & 238 & $121(50,8)$ & $117(49,2)$ & 1 & \\
\hline Negativa & 201 & $83(41,3)$ & $118(58,7)$ & $1,06(1,01-1,13)$ & 0,044 \\
\hline \multicolumn{6}{|c|}{ Acesso aos serviços de saúde da UAPS nos últimos 12 meses } \\
\hline Sim & 375 & $173(46,1)$ & $202(53,9)$ & 1 & \\
\hline Não & 62 & $30(48,4)$ & $32(51,6)$ & $0,98(0,90-1,07)$ & 0,744 \\
\hline \multicolumn{6}{|c|}{ Recebimento de visita domiciliar } \\
\hline Mensalmente & 152 & $68(44,7)$ & $84(55,3)$ & 1 & \\
\hline Ocasionalmente & 287 & $136(47,4)$ & $151(52,6)$ & $0,98(0,92-1,04)$ & 0,595 \\
\hline \multicolumn{6}{|c|}{ Posse de plano de saúde particular } \\
\hline Sim & 110 & $63(57,3)$ & $47(42,7)$ & 1 & \\
\hline Não & 382 & $140(45,7)$ & $188(57,3)$ & $1,10(1,02-1,18)$ & 0,009 \\
\hline \multicolumn{6}{|c|}{ Participação em grupo de direitos sexuais e reprodutivos } \\
\hline Sim & 39 & $21(53,8)$ & $18(46,2)$ & 1 & \\
\hline Não & 399 & $182(45,6)$ & $217(54,4)$ & $1,05(0,94-1,18)$ & 0,337 \\
\hline
\end{tabular}

LFS: letramento funcional em saúde; RP: razão de prevalência; IC: intervalo de confiança; UAPS: Unidade de Atenção Primária à Saúde. * As oscilações do tamanho da amostra são justificadas pela ausência de informação. ${ }^{\dagger}$ Sentir-se à vontade para conversar com pelo menos um amigo ou familiar

Para a análise multivariada, optou-se pela classificação socioeconômica segundo os critérios da ABEP, sendo excluídas as variáveis "renda per capita" e "posse de plano de saúde particular" por serem altamente correlacionadas. No modelo final de regressão, o nível baixo de LFS associou-se à idade superior aos 40 anos ( $R P=1,18 ; \mathrm{IC} 95 \%: 1,07-1,31)$, ao grau de instrução inferior ao ensino médio completo $(\mathrm{RP}=1,26$; IC $95 \%: 1,15-1,38)$, à baixa renda $(\mathrm{RP}=1,13$; IC 95\%: 1,04-1,23) e à autodeclaração da cor parda ou preta ( $R P=1,06$; IC 95\%: 1,01-1,12) (Tabela 3). 
Tabela 3. Modelo final de regressão de Poisson, com razões de prevalência bruta e ajustada, intervalos de confiança de $95 \%$ e valores p, para as associações das variáveis selecionadas com o nível baixo de letramento funcional em saúde (LFS) em mulheres atendidas pela Estratégia de Saúde da Família, Juiz de Fora, MG, 2015-2016

\begin{tabular}{|c|c|c|c|c|}
\hline \multirow[b]{2}{*}{ Variável } & \multicolumn{4}{|c|}{ Nível baixo de LFS } \\
\hline & $\begin{array}{l}\text { RP bruta } \\
\text { (IC 95\%) }\end{array}$ & $\mathbf{p}$ & $\begin{array}{l}\text { RP ajustada } \\
\quad \text { (IC 95\%) }\end{array}$ & $\mathbf{p}$ \\
\hline \multicolumn{5}{|l|}{ Faixa etária } \\
\hline 25 a 29 anos & 1 & & 1 & \\
\hline 30 a 39 anos & $1,11(0,99-1,23)$ & 0,062 & $1,09(0,98-1,21)$ & 0,086 \\
\hline 40 a 49 anos & $1,26(1,13-1,39)$ & $<0,001$ & $1,18(1,07-1,31)$ & 0,001 \\
\hline 50 a 59 anos & $1,44(1,31-1,59)$ & $<0,001$ & $1,29(1,16-1,43)$ & $<0,001$ \\
\hline$\geq 60$ anos & $1,52(1,37-1,70)$ & $<0,001$ & $1,41(1,27-1,57)$ & $<0,001$ \\
\hline \multicolumn{5}{|l|}{ Grau de instrução } \\
\hline Ensino médio completo ou mais & 1 & & 1 & \\
\hline $\begin{array}{l}\text { Ensino fundamental completo ou } \\
\text { médio incompleto }\end{array}$ & $1,31(1,22-1,41)$ & $<0,001$ & $1,22(1,13-1,32)$ & $<0,001$ \\
\hline $\begin{array}{l}\text { Analfabeta ou ensino fundamental } \\
\text { incompleto }\end{array}$ & $1,45(1,35-1,56)$ & $<0,001$ & $1,26(1,15-1,38)$ & $<0,001$ \\
\hline \multicolumn{5}{|l|}{ Situação socioeconômica ${ }^{\S}$} \\
\hline Média/alta & 1 & & 1 & \\
\hline Baixa & $1,24(1,13-1,35)$ & $<0,001$ & $1,13(1,04-1,23)$ & 0,002 \\
\hline \multicolumn{5}{|l|}{ Cor da pele autodeclarada } \\
\hline Branca & 1 & & 1 & \\
\hline Parda/negra & $1,04(0,97-1,11)$ & 0,228 & $1,08(1,02-1,14)$ & 0,006 \\
\hline
\end{tabular}

LFS: letramento funcional em saúde; RP, razão de prevalência; IC, intervalo de confiança. ${ }^{5}$ Renda média/alta: classes A, B1 e B2; renda baixa: classes $\mathrm{C} 1, \mathrm{C} 2$ e D/E'

\section{DISCUSSÃO}

No presente estudo, verificou-se o baixo LFS em 53,5\% das mulheres avaliadas. Considerando os estudos realizados no Brasil que utilizaram o B-TOFHLA, essa prevalência é semelhante ao resultado obtido por Passamai ${ }^{10}$ em Fortaleza (53,8\%), com usuários da Atenção Primária à Saúde. Em relação aos pacientes recrutados no nível secundário de atenção, Sampaio et al. ${ }^{12}$ e Santos \& Portella ${ }^{13}$ encontraram maiores prevalências de baixo LFS $(65,9 \%$ e 73,7\%, respectivamente) em pacientes diabéticos da região Nordeste do País. Ao avaliarem indivíduos recrutados no nível terciário de atenção, Carthery-Goulart et al. ${ }^{9}$, assim como Apolinário et al. ${ }^{14,15}$, encontraram prevalências inferiores de baixo LFS $(23,4 \%, 31,7 \%$ e $31,7 \%$, respectivamente) em indivíduos hospitalizados da Grande São Paulo. O nível de LFS identificado nos estudos conduzidos no Brasil apresentou extensa amplitude, oscilando de $31,7 \%$ a $73,7 \%$, de forma que a menor prevalência foi encontrada em São Paulo e a maior em Belém, o que pode refletir a disparidade nacional de acesso à educação e a distribuição heterogênea da escolaridade no País ${ }^{16}$.

As mulheres mais velhas apresentaram probabilidade entre $20 \%$ e $40 \%$ maior para o baixo LFS, associação também demostrada em outros estudos ${ }^{10,17,18}$. À medida que a idade avança, diminui a capacidade de realizar tarefas cognitivas que exigem processamento de informações. Os adultos mais velhos tendem a ter mais dificuldade em completar tarefas que 
exigem raciocínio ou inferência das informações que lhes são apresentadas, o que sugere maior prevalência de baixo LFS em faixas etárias elevadas ${ }^{19}$.

Por outro lado, as mulheres mais velhas apresentaram menor escolaridade, quando comparadas às mais jovens. Isso pode ser decorrente da transição educacional vivenciada no Brasil, cujos investimentos públicos progressivos nesse setor propiciaram um maior acesso à escola, melhorando o nível educacional da população nas últimas décadas ${ }^{16}$.

Mulheres com grau de instrução inferior ao ensino médio completo apresentaram 25\% maior probabilidade para o baixo LFS. Apesar da relação entre escolaridade e LFS já ter sido demonstrada 9, 12-15,17,20-26, é válido ressaltar que o indicador "anos completos de estudo" pode não refletir o grau de alfabetização. O nível de LFS traduz a capacidade do indivíduo para adquirir conhecimentos e lidar com as demandas sociais, principalmente no contexto da saúde ${ }^{10}$. Além disso, o nível educacional não garante um LFS adequado, uma vez que usuários com alta escolaridade podem exibir dificuldades com terminologias e procedimentos relativos ao contexto da saúde 27,28 .

Mulheres de baixa renda foram $13 \%$ mais susceptíveis ao baixo LFS, quando comparadas às de média e alta renda, corroborando outros resultados ${ }^{29-31}$. A renda é o indicador de status social que mede diretamente o nível de vida material, estando interligada com outros indicadores, como emprego, tipo de trabalho e acesso a serviços de promoção da saúde. Indivíduos em menor estrato socioeconômico usualmente têm maiores dificuldades para acessarem serviços e receberem informações de saúde, o que ocasiona piores níveis de LFS e desfechos clínicos ${ }^{30}$.

As mulheres que se autodeclararam pardas ou pretas tiveram uma probabilidade $8 \%$ maior de exibirem baixo LFS, resultado que já foi demonstrado em outras investigações ${ }^{14,17,29,32-34}$. Pode-se dizer que a cor da pele autodeclarada reflete a confluência de fatores biológicos, geográficos, culturais, econômicos, políticos e legais ${ }^{35}$. As barreiras geradas pelas desigualdades raciais são determinantes para o acesso e a utilização dos serviços de saúde ${ }^{36}$. Embora o Brasil possua um sistema de saúde público, universal, igualitário e equânime ${ }^{37}$, com avanços acerca de políticas de promoção à igualdade racial, ainda persistem desigualdades nas estruturas sociais e no acesso aos serviços de saúde, gerando reflexos nas condições de vida e no processo saúde-doença das mulheres negras ${ }^{38}$, circunstância que impacta diretamente no nível de LFS.

Cabe mencionar que a prevalência de baixo LFS deste estudo, apesar de alta, deve ser interpretada com cautela, pois se trata de uma amostra previamente sensibilizada quanto à promoção da saúde e mudanças no estilo de vida.

As associações verificadas entre o LFS e as variáveis idade, grau de instrução, situação socioeconômica e cor da pele reforçam o princípio de que o LFS é composto por estruturas sociais mais complexas do que as descritas apenas pela educação ${ }^{6}$. Mulheres mais velhas, de baixa renda, que não concluíram o ensino médio ou que pertencem a grupos étnicos considerados minoritários têm maiores probabilidades de apresentarem baixo LFS ${ }^{39}$.

Considera-se que aqueles com LFS inadequado ou marginal terão dificuldade na leitura, na compreensão e na interpretação de orientações para o cuidado com a saúde, estando mais propícios não apenas ao uso incorreto de medicações prescritas, mas também ao abandono do plano de cuidados ${ }^{1-4}$. Por isso, torna-se emergente alertar os profissionais da saúde para a necessidade de se adequar os discursos utilizados nos atendimentos ao nível de compreensão dos usuários. Ademais, a expansão de políticas públicas voltadas para a promoção da saúde, que contribuam para diminuir as iniquidades, além do estímulo à alfabetização e incentivo a atividades de educação em saúde podem constituir caminhos institucionais para a melhora do nível de LFS da população.

\section{AGRADECIMENTOS}

Os autores agradecem ao Núcleo de Assessoria, Treinamento e Estudos em Saúde - NATES da Universidade Federal de Juiz de Fora - UFJF, à Prefeitura de Juiz de Fora, aos profissionais das duas Unidades de Atenção Primária à Saúde avaliadas, ao Instituto de Medicina Social - IMS da Universidade do Estado do Rio de Janeiro - UERJ, e, sobretudo, às mulheres participantes, sem as quais este estudo não existiria. 


\section{REFERÊNCIAS}

1. World Health Organization. Health literacy: the solid facts [Internet]. Copenhagen: World Health Organization; 1998 [citado em 2017 Jun 8]. Disponível em: http://www.euro.who.int/_data/assets/ pdf_file/0008/190655/e96854.pdf

2. Sorensen K, Van den Broucke S, Fullam J, Doyle G, Pelikan J, Slonska Z, et al. Health literacy and public health: A systematic review and integration of definitions and models. BMC Public Health. 2012;12(1):80. http://dx.doi.org/10.1186/1471-2458-12-80. PMid:22276600.

3. Weiss BD. Health literacy and patient safety: help patients understand. $2^{\mathrm{a}}$ ed. Chicago: American Medical Association Foundation; 2007.

4. McCarthy DM, Waite KR, Curtis LM, Engel KG, Baker DW, Wolf MS. What did the doctor say? Health literacy and recall of medical instructions. Med Care. 2012;50(4):277-82. http://dx.doi.org/10.1097/ MLR.0b013e318241e8e1. PMid:22411440.

5. Brasil. Ministério da Saúde. Secretaria de Atenção à Saúde. Departamento de Atenção Básica. Política Nacional de Atenção Básica [Internet]. Brasília: Ministério da Saúde; 2012 [citado em 2017 Abr 20]. Disponível em: http://189.28.128.100/dab/docs/publicacoes/geral/pnab.pdf

6. Carmo Rocha J, Bustamante Teixeira MT, Azevedo e Silva G, Castro Dias K, Salim Miranda Duque ML. Prevalence of prehypertension and associated factors in women. Invest Educ Enferm. 2014;32(3):471-9. http://dx.doi.org/10.17533/udea.iee.v32n3a12. PMid:25504413.

7. Brasil. Ministério da Saúde. Projeto Olhar Brasil: triagem de acuidade visual: Manual de orientação [Internet]. Brasília: Ministério da Saúde; 2008 [citado em 2015 Apr 20]. Disponível em: http://portaldoprofessor.mec. gov.br/storage/materiais/0000015497.pdf

8. Baker DW, Williams MV, Parker RM, Gazmararian JA, Nurss J. Development of a brief test to measure functional health literacy. Patient Educ Couns. 1999;38(1):33-42. http://dx.doi.org/10.1016/S07383991(98)00116-5. PMid:14528569.

9. Carthery-Goulart MT, Anghinah R, Areza-Fegyveres R, Bahia VS, Brucki SMD, Damin A, et al. Performance of a Brazilian population on the test of functional health literacy in adults. Rev Saude Publica. 2009;43(4):6318. http://dx.doi.org/10.1590/S0034-89102009005000031. PMid:19488667.

10. Passamai MPB. Letramento funcional em saúde de adultos no contexto do Sistema Único de Saúde: um caminho para a promoção da saúde e prevenção de doenças crônicas não transmissíveis [tese]. Fortaleza: Universidade Estadual do Ceará; 2012.

11. Associação Brasileira de Empresas de Pesquisa. Critério de Classificação Econômica Brasil [Internet]. 2015 [citado em 2015 nov 20]. Disponível em: http://www.abep.org.br/site/

12. Sampaio HAC, Carioca AAF, Sabry MOD, Santos PM, Coelho MAM, Passamai MPB. Letramento em saúde de diabéticos tipo 2: fatores associados e controle glicêmico. Cien Saude Colet. 2015;20(3):865-74. http:// dx.doi.org/10.1590/1413-81232015203.12392014. PMid:25760126.

13. Santos MI, Portella MR. Conditions of functional health literacy of an elderly diabetics group. Rev Bras Enferm. 2016;69(1):144-52. PMid:26871229.

14. Apolinario D, Mansur LL, Carthery-Goulart MT, Brucki SM, Nitrini R. Detecting limited health literacy in Brazil: development of a multidimensional screening tool. Health Promot Int. 2014;29(1):5-14. http:// dx.doi.org/10.1093/heapro/dat074. PMid:24179154.

15. Apolinario D, Mansur LL, Carthery-Goulart MT, Brucki SM, Nitrini R. Cognitive predictors of limited health literacy in adults with heterogeneous socioeconomic backgrounds. J Health Psychol. 2015;20(12):1613-25. http://dx.doi.org/10.1177/1359105313520337. PMid:24496057.

16. Organisation for Economic Co-Operation and Development. Education at a glance: OECD indicators [Internet]. Paris: OECD; 2015 [citado em 2017 Apr 21]. Disponível em: https://www.oecd.org/brazil/ Education-at-a-glance-2015-Brazil-in-Portuguese.pdf

17. Castro $\mathrm{SH}$, Brito GN, Gomes MB. Health literacy skills in type 2 diabetes mellitus outpatients from an university-affiliated hospital in Rio de Janeiro, Brazil. Diabetol Metab Syndr. 2014;6(1):126. http://dx.doi. org/10.1186/1758-5996-6-126. PMid:25960771.

18. Kobayashi LC, Wardle J, Wolf MS, von Wagner C. Aging and functional health literacy: a systematic review and meta-analysis. J Gerontol B Psychol Sci Soc Sci. 2016;71(3):445-57. http://dx.doi.org/10.1093/geronb/ gbu161. PMid:25504637.

19. Baker DW, Gazmararian JA, Sudano J, Patterson M. The association between age and health literacy among elderly persons. J Gerontol B Psychol Sci Soc Sci. 2000;55(6):S368-3674. http://dx.doi.org/10.1093/ geronb/55.6.S368. PMid:11078114. 
20. Oliveira MO, Porto CS, Brucki SMD. S-TOFHLA in mild Alzheimer's disease and mild cognitive impairment patients as a measure of functional literacy: preliminary study. Dement Neuropsychol. 2009;3(4):291-8. http://dx.doi.org/10.1590/S1980-57642009DN30400005. PMid:29213642.

21. Brucki SMD, Mansur LL, Carthery-Goulart MT, Nitrini R. Formal education, health literacy and minimental state examination. Dement Neuropsychol. 2011;5(1):26-30. http://dx.doi.org/10.1590/S198057642011DN05010005. PMid:29213716.

22. Apolinario D, Braga Rde C, Magaldi RM, Busse AL, Campora F, Brucki S, et al. Short assessment of health literacy for Portuguese-speaking adults. Rev Saude Publica. 2012;46(4):702-11. http://dx.doi.org/10.1590/ S0034-89102012005000047. PMid:22782124.

23. de Oliveira MO, Nitrini R, Brucki SM. The S-TOFHLA as a measure of functional literacy in patients with mild Alzheimer's disease or mild cognitive impairment. Arch Clin Neuropsychol. 2014;29(3):269-77. http:// dx.doi.org/10.1093/arclin/act120. PMid:24503948.

24. Morris NS, Maclean CD, Littenberg B. Change in health literacy over 2 years in older adults with diabetes. Diabetes Educ. 2013;39(5):638-46. http://dx.doi.org/10.1177/0145721713496871. PMid:23963099.

25. Reisi M, Javadzade SH, Heydarabadi AB, Mostafavi F, Tavassoli E, Sharifirad G. The relationship between functional health literacy and health promoting behaviors among older adults. J Educ Health Promot. 2014;3(1):119. http://dx.doi.org/10.4103/2277-9531.145925. PMid:25540792.

26. Heijmans M, Waverijn G, Rademakers J, van der Vaart R, Rijken M. Functional, communicative and critical health literacy of chronic disease patients and their importance for self-management. Patient Educ Couns. 2015;98(1):41-8. http://dx.doi.org/10.1016/j.pec.2014.10.006. PMid:25455794.

27. Institute of Medicine. Health literacy: a prescription to end confusion [Internet]. Washington, DC: National Academies Press; 2004 [citado em 2017 Abr 8]. Disponível em: https://www.nap.edu

28. United States Department of Health and Human Services. America's health literacy: why we need accessible health information [Internet]. Washington, DC; 2008 [citado em 2017 Abr 15]. Disponível em: http://www. health.gov/communication/literacy/issuebrief/\#lower

29. Souza JG, Apolinario D, Magaldi RM, Busse AL, Campora F, Jacob-Filho W. Functional health literacy and glycaemic control in older adults with type 2 diabetes: A cross-sectional study. BMJ Open. 2014;4(2):1-8. http://dx.doi.org/10.1136/bmjopen-2013-004180. PMid:24525392.

30. Toçi E, Burazeri G, Kamberi H, Jerliu N, Sorensen K, Brand H. Socio-economic correlates of functional health literacy among patients of primary health care in Kosovo. Public Health. 2014;128(9):842-8. http://dx.doi. org/10.1016/j.puhe.2014.06.009. PMid:25129227.

31. Becerra MB, Becerra BJ, Daus GP, Martin LR. Determinants of low health literacy among Asian-American and Pacific Islanders in California. J Racial Ethn Health Disparities. 2015;2(2):267-73. http://dx.doi.org/10.1007/ s40615-015-0092-0. PMid:26863342.

32. Kaphingst KA, Goodman M, Pyke O, Stafford J, Lachance C. Relationship between self-reported racial composition of high school and health literacy among community health center patients. Health Educ Behav. 2012;39(1):35-44. http://dx.doi.org/10.1177/1090198111406538. PMid:21636703.

33. Mantwill S, Monestel-Umaña S, Schulz PJ. The relationship between health literacy and health disparities: a systematic review. PLoS One. 2015;10(12):1-22. http://dx.doi.org/10.1371/journal.pone.0145455. PMid:26698310.

34. Levy H, Janke A. Health literacy and access to care. J Health Commun. 2016;21(Suppl 1):43-50. http:// dx.doi.org/10.1080/10810730.2015.1131776. PMid:27043757.

35. Santos JAF. Desigualdade racial de saúde e contexto de classe no brasil. Dados. 2011;54(1):5-40. http:// dx.doi.org/10.1590/S0011-52582011000100001.

36. Batista LE, Werneck J, Lopes F. Saúde da população negra. 2a ed. Brasília: Associação Brasileira de Pesquisadores Negros; 2012 [citado em 2017 Abr 25]. Disponível em: http://bvsms.saude.gov.br/bvs/ publicacoes/saude_populacao_negra.pdf

37. Brasil. Lei n 8.080 , de 19 de setembro de 1990. Dispõe sobre as condições para a promoção, proteção e recuperação da saúde, a organização e o funcionamento dos serviços correspondentes e dá outras providências. Diário Oficial da União, Brasília, 20 de setembro de 1990; Seção 1. p. 18055-9.

38. Brasil. Ministério da Saúde. Associação Brasileira de Pesquisadores Negros. Saúde da população negra [Internet]. Brasília: Ministério da Saúde; 2012 [citado em 2016 Jun 28]. Disponível em: http://bvsms.saude. gov.br/bvs/publicacoes/saude_populacao_negra.pdf

39. Nutbeam D. Defining and measuring health literacy: what can we learn from literacy studies? Int J Public Health. 2009;54(5):303-5. http://dx.doi.org/10.1007/s00038-009-0050-x. PMid:19641847. 